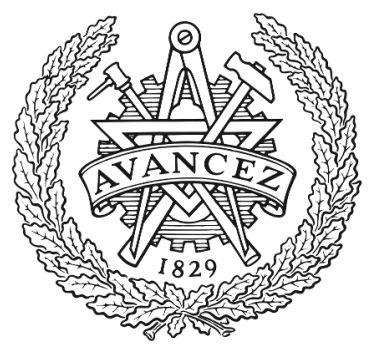

CHALMERS

UNIVERSITY OF TECHNOLOGY

\title{
A Shared-Path Shared-Compute Planning Strategy for a Resilient Hybrid C-RAN
}

Downloaded from: https://research.chalmers.se, 2023-04-26 12:46 UTC

Citation for the original published paper (version of record):

Lashgari, M., Wosinska, L., Monti, P. (2019). A Shared-Path Shared-Compute Planning Strategy for a Resilient Hybrid C-RAN. International Conference on Transparent Optical Networks, 2019-July. http://dx.doi.org/10.1109/ICTON.2019.8840404

N.B. When citing this work, cite the original published paper. 


\title{
A Shared-Path Shared-Compute Planning Strategy for a Resilient Hybrid C-RAN
}

\author{
Maryam Lashgari, Lena Wosinska, and Paolo Monti \\ Department of Electrical Engineering, Chalmers University of Technology, Gothenburg, Sweden \\ E-mails: \{maryaml, wosinska, mpaolo\}@chalmers.se
}

\begin{abstract}
One key challenge in 5G networks is to guarantee the survivability of services in the event of failures. This paper focuses on the hybrid cloud radio access network (H-CRAN) architecture. The proposed strategy guarantees survivability in the presence of failures affecting nodes/links in the midhaul segment (i.e., connecting the radio aggregation unit (RAU) nodes to their respective radio cloud center (RCC) nodes) as well as compute resources (i.e., servers) in the RCC nodes.

In the envisioned strategy each RAU node is connected to a primary and a backup RCC node (i.e., with backup compute resources) via two node disjoint connectivity paths in the midhaul. The proposed strategy, called Shared-Path Shared-Compute Planning (SPSCP), lowers the overall design cost by trying to share as much as possible backup connectivity and compute resources among RAU nodes. This is made possible by introducing a shareability metric early into the RCC node selection process so that the chance of sharing backup resources is maximized. Simulation results show that the SPSCP strategy can lead to up to $28 \%$ cost savings when compared to conventional resilient design strategies.
\end{abstract}

Keywords: 5G, resiliency, network design, hybrid CRAN (H-CRAN), resource sharing, node failure, link failure, server failure, fronthaul, midhaul

\section{INTRODUCTION}

The hybrid cloud radio access network (H-CRAN) architecture is a promising option to meet the 5G service requirements in terms of high quality of service (QoS), quality of experience (QoE), and survivability [1]. An H-CRAN architecture consists of three tiers: remote radio units (RRUs), radio aggregation units (RAUs), and radio cloud centers (RCCs) [2]. More specifically, some of the baseband processing functions are implemented in the RAU node, while the rest of the functions are incorporated at the RCC node [3]. The section of the network connecting RRUs and the RAU nodes, referred to as the fronthaul segment, can be based either on wired (e.g., optical and/or packet based) or wireless (e.g., millimeter wave) technologies. The section of the network connecting RAU and RCC nodes is normally referred to as the midhaul segment. The Common Public Radio Interface (CPRI/eCPRI) [4] and the Next Generation Fronthaul Interface (NGFI) [3] are two possible options to transmit data from the RRUs to the $5 \mathrm{G}$ core. One interesting thing to note is that NGFI advocates the use of mesh and packet-based networks (i.e., instead of using just point-to-point or point-to-multipoint optical links) for the midhaul segment [5].

The number of users affected by a failure depends on where the failure happens within the H-CRAN. The RAU node is responsible for serving all the RRUs connected to it, while the RCC node provides services for the area covered by all its downstream RAU nodes. Failures might affect an H-CRAN in various ways, depending on the type and on the failure location. For example, a failure might affect the RCC node partly or completely. The same might happen to RAU nodes. In addition, failures might affect RRUs and/or links/nodes in the fronthaul and in the midhaul network. Given that a few RCC nodes cover a large number of users, the design of a resilient H-CRAN needs to consider RCC node failures in addition to the planning of resilient midhaul network [2], [3].

A number of works in the literature studied the resilient design problem for centralized RAN architectures [6]-[9]. The authors in [6] solve the survivable baseband unit (BBU) hotel placement problem in a C-RAN to guarantee resiliency from a single BBU hotel failure. Their algorithm consists of two steps. In the first one, the algorithm decides the placement of a minimum number of primary and backup BBU hotels. In the second step, the algorithm tries to maximize the amount of backup BBU ports that could be shared among RRUs. The work in [7] considers a C-RAN deployment over a 5G optical aggregation network and proposes three approaches for the combined survivable BBU hotel placement and traffic routing problem. More specifically, the work proposes an ILP formulation for the following three subproblems: dedicated path protection, dedicated BBU protection, and dedicated BBU and path protection. In [8], the authors derive an ILP formulation to select the best BBU in terms of failure probability and energy efficiency, while maximizing the amount of traffic processed by the C-RAN.

As it becomes evident from the previous paragraph, most of the interest is related to the C-RAN case, while the problem of designing a resilient H-CRAN architecture has not been considered at all in the existing literature. Additionally, most of the resilient C-RAN design solutions solve the problem by duplicating the primary resources, an approach which is not cost efficient. The few approaches that allow sharing of backup resources (e.g., [6]) consider only the sharing of BBU ports in BBU hotels. On the other hand, sharing the backup connectivity and compute resources used to protect the midhaul network and the RCC nodes has the 


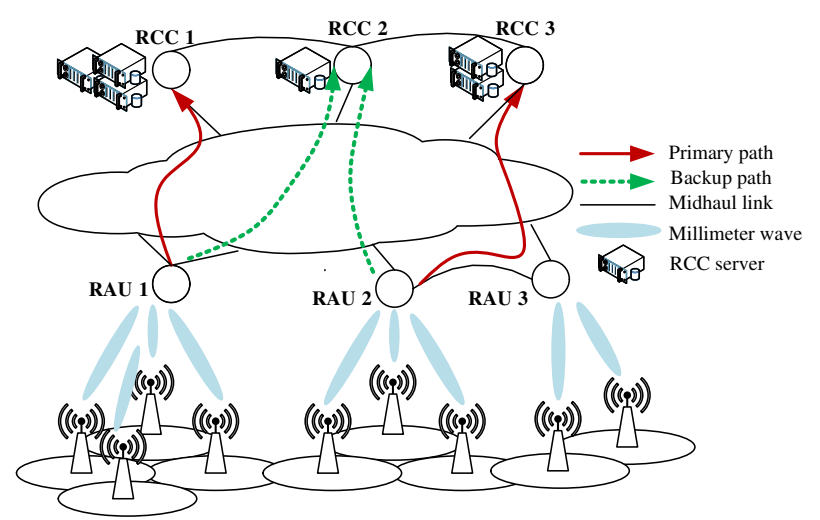

Figure 1: An example of an H-CRAN architecture with a mesh midhaul network and a millimeter wave fronthaul.

potential to reduce considerably the H-CRAN deployment cost. Also, the few strategies that leverage on sharing backup resources usually work in a sequential fashion. In a first stage, primary and backup BBU locations are chosen without considering the impact that a given choice might have on the possibility to share backup resources. Only in a second stage the number of shared backup resources (e.g., BBU ports) is minimized. On the other hand, the number of backup resources that could potentially be shared might increase if resource shareability is considered while deciding on the placement of primary and backup resources.

This paper presents a strategy that guarantees the survivability of any service running over an H-CRAN in the presence of failures affecting the midhaul nodes/links and servers in the RCC nodes. For each RAU in the HCRAN, the proposed strategy (called Shared-Path Shared-Compute Planning (SPSCP)) decides on the placement of the primary and backup RCC nodes as well as on the routes of their respective primary and backup paths in such a way that the sharing of the compute (i.e., in the RCC nodes) and the connectivity resources (i.e., in the midhaul network) are maximized. Simulation results show that SPSCP is able to outperform those resilient design strategies using only dedicated backup resources (i.e., $28 \%$ in cost gain), or those where the shareability metric is not considered while deciding the placement of the primary and backup resources (i.e., $23 \%$ in cost gain).

The rest of the paper is organized as follows. Section II presents the system architecture and the use case description, while Section III discusses the proposed design algorithm. Simulation results are analyzed in Section IV. Finally, conclusions are drawn in Section V.

\section{System Architecture and USe Case}

This work considers an H-CRAN architecture with a mesh packet over wavelength division multiplexing (WDM) network as the midhaul. The RAU nodes are deployed in a subset of the midhaul network nodes and act as the edge cloud. Figure 1 presents the architecture considered for this work. We assume an eCPRI interface for the connections between RCC and RAU nodes, as well as for connecting RAU nodes with RRUs.

Let's assume that $\mathcal{N}$ represents the set of nodes in the midhaul network, while the set of RAU nodes is represented by $\mathcal{A}$. As a result, the set of possible locations to place the RCC node is given by $\mathcal{C}=\mathcal{N} \backslash \mathcal{A}$. Consider $s_{i}$ to be the number of servers RAU node $i$ requires in the RCC node for baseband processing functionalities. As a result, $\mathcal{S}=\left\{s_{i}\right\}, \forall i \in \mathcal{A}$ is the set describing the number of servers required by each one of the RAU nodes in the H-CRAN. The value of $s_{i}$ depends on the number of RRUs that are connected to and served by RAU node $i$.

In the current work we consider a single failure assumption. Failures can affect a link or a node in the midhaul network, as well as a server in the RCC nodes. In addition, we assume that it is also possible to lose an entire RCC node due to a (single) catastrophic event (e.g., fire in the central office). Failures affecting RRUs and RAU nodes are outside the scope of this work. The assumption is that the mobile operator has already planned for these scenarios with dual-homed RRUs, while the effects of the RRU failure can be managed according to the operator handover policy.

The aim of this work is to design a resilient H-CRAN architecture. This translates into by assigning, for each deployed RAU node, one primary and one backup RCC node location to which the RAU node will have to be connected via two node disjoint paths in the midhaul network. The above objective needs to be achieved while minimizing the total cost of the H-CRAN, defined as follows:

$$
C=N_{R C C} \cdot C_{R C C}+N_{C o n n} \cdot C_{C o n n}+N_{S e r} \cdot C_{S e r},
$$

where $N_{R C C}$ is the number of RCC nodes deployed in the H-CRAN; $C_{R C C}$ is the cost of deploying one RCC node; $N_{C o n n}$ is the total number of connectivity units (over the all links) used in the midhaul network; $C_{C o n n}$ 


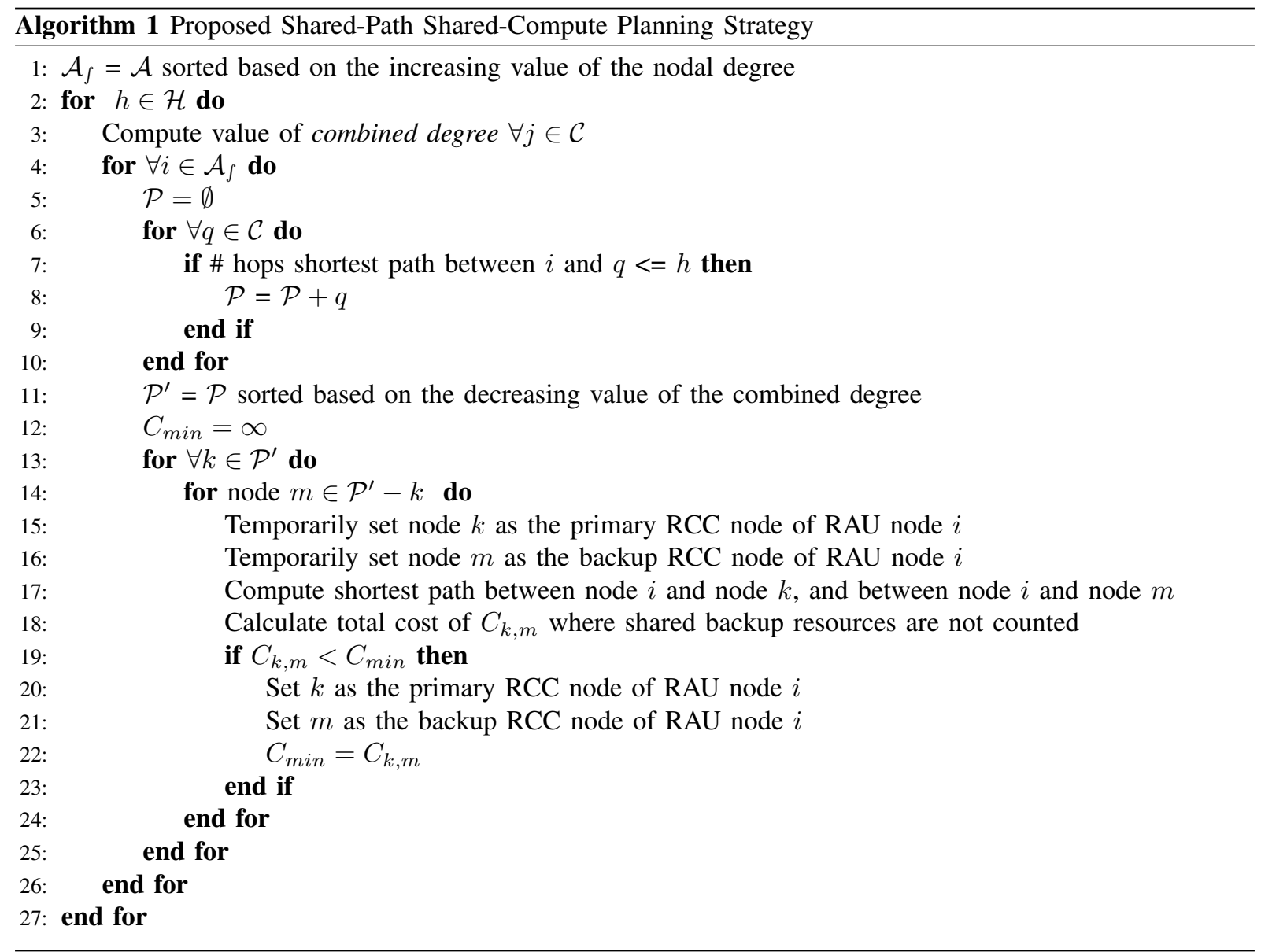

is cost of one connectivity unit (per midhaul link); $N_{\text {Ser }}$ is the total number of server units to be deployed in all the RCC nodes; and $C_{S e r}$ is the cost of a server unit. In order to account for the latency requirements of a service running over the H-CRAN we assume that the connectivity path between the RAU node and its primary and backup RCC nodes cannot exceed a given number of hops $h$, where $h \in \mathcal{H}$.

One possible way to reduce the total design cost is to encourage sharing of backup resources. In this work we allow for the sharing of connectivity resources in the midhaul network and of the server units in the RCC nodes. More specifically, backup connectivity resources can be shared between two (or more) RAU nodes if and only if the following two conditions are satisfied: (i) their primary connectivity paths are node disjoint, and (ii) their primary servers reside in different RCC nodes. We refer to these two conditions as connectivity sharing conditions. Similarly, two (or more) RAU nodes can share backup server units in the same RCC node if and only if the following two conditions are satisfied: $(i)$ their primary server units are placed in different RCC nodes, and (ii) their primary connectivity paths in the midhaul network are node disjoint. We refer to these two conditions as server sharing conditions.

It is possible to maximize the benefits of sharing backup resources by strategically deciding on the placing the primary and backup RCC nodes and on the route of primary and backup connectivity paths in the midhaul. The next section introduces a planning strategy with this specific objective in mind.

\section{The Shared-Path Shared-Compute Planning Strategy}

This section describes the proposed resilient design strategy referred to as Shared-Path Shared-Compute Planning (SPSCP).

For any given RAU node, the intuition behind the approach is to list all the possible locations for the primary and backup RCC nodes and rate them in terms of their cost value, i.e., as defined by (1). The solution with the minimum cost value is then chosen and the next RAU node is considered. The procedure finishes when all RAU nodes are assigned a primary and a backup RCC node and connected via their respective primary and backup connectivity paths over the midhaul. The details of the strategy are summarized in Algorithm 1.

In step 1 , set $\mathcal{A}_{\int}$ is derived from set $\mathcal{A}$ by sorting its elements in the increasing order of their nodal degree. Step 3 defines a parameter called the combined degree. For each midhaul node $j \in \mathcal{C}$ this parameter is defined as the sum of the value of the nodal degree of $j$ and the number of RAU nodes that are within $h$ hops from $j$. The intuition behind using this parameter is to identify those midhaul nodes that are more likely to serve a 


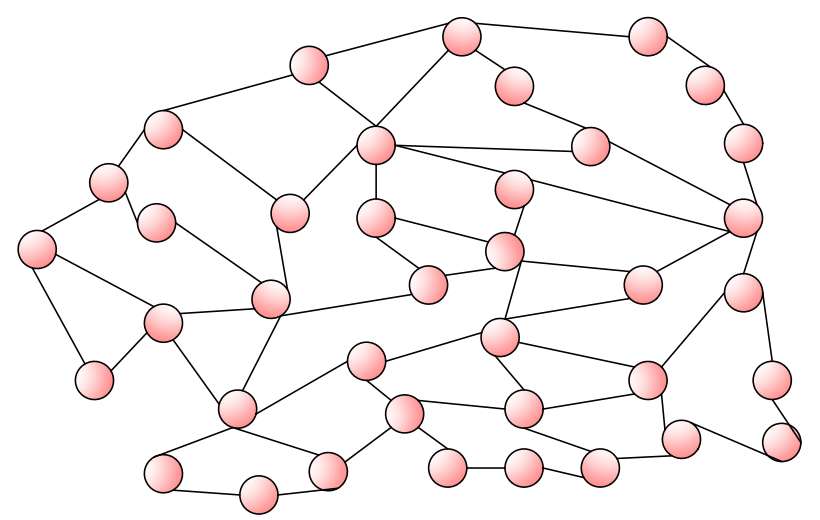

Figure 2: The mesh midhaul network with 38 transport nodes.

high number of RAU nodes if chosen as the RCC node location, thus improving the overall cost savings of the designed solution. In steps 4-10, the midhaul nodes whose shortest path is within $h$ hops from RAU node $i$ are put in set $\mathcal{P}$. Then, set $\mathcal{P}^{\prime}$ is derived from $\mathcal{P}$ by sorting its elements based on the decreasing value of their combined degree (step 11). In steps 13-24, given the RAU node $i$, we compute the value of total network cost (i.e., $C_{k, m}$ ) for all the different primary/backup RCC node options and we then choose the one which brings the lowest cost. More specifically, for each candidate location for the primary RCC node $k \in \mathcal{P}^{\prime}$ the algorithm checks all the possible locations for the backup RCC node $m \in \mathcal{P}^{\prime}-k$ and it then computes the resulting total network cost value $C_{k, m}$ (step 15). The route of the primary and backup paths in the midhaul network are computed using the shortest path. If backup resources can be shared in either the backup RCC node or over the midhaul network (i.e., according to the server sharing and connectivity sharing conditions defined in Sec. 2) they are not counted while computing the value of $C_{k, m}$. If the value of $C_{k, m}$ is less than the minimum cost value found so far (i.e., $C_{\text {min }}$ ), then node $k$ and node $m$ are selected as the primary and backup RCC nodes for RAU node $i$, respectively. The procedure is repeated for all nodes $i$ in set $\mathcal{A}_{f}$.

\section{Performance evaluation}

In this section, we evaluate the performance of the SPSCP strategy via simulations. We assume a transport network with 38 nodes as the midhaul network (Fig. 2). It is assumed that $40 \%$ of the midhaul nodes are RAU nodes, while their location is chosen randomly from a uniform distribution within the range $[1,38]$.

We assume a given baseband functional split at the interface between the RAU and the RCC node as well as at the interface between the RRU and the RAU node. Split options 2 and 7 are implemented in the midhaul and in the fronthaul, respectively [10]. According to the eCPRI specification and based on the selected split points, the capacity requirements over the midhaul are $4016 \mathrm{Mb} / \mathrm{s}$ and $3024 \mathrm{Mb} / \mathrm{s}$ for down link and up link transmission, respectively. For simplicity, in this work we only consider the up link direction. Similar considerations can also be made for the design and dimensioning of the down link direction. Set $\mathcal{S}$ is populated $(\forall i \in \mathcal{A})$ using a uniform distribution in the range $[5,15]$.

In the simulations, the value of $h \in \mathcal{H}$ is varied between 2 and 10. The cost of deploying one RCC node is assumed to be $C_{R C C}=100$ cost unit [CU]. In order to reflect the fact that deploying the RCC node is more expensive than a server unit or one connectivity unit (i.e., equal to $100 \mathrm{~Gb} / \mathrm{s}$ ), their cost per unit is chosen much lower than the RCC node cost. In addition, it is assumed that the midhaul network has no limitation on the number of connectivity unit resources.

The performance of the proposed SPSCP strategy is evaluated against three benchmark algorithms. In the first one, referred to as resource duplication $(R D)$, each RAU node is connected to a primary and a backup RCC node via two node disjoint connectivity paths in the midhaul such that the cost function described in (1) is minimized, but no sharing of backup resources is allowed. The second benchmark strategy, i.e., preliminary resource sharing $(P R S)$, aims to improve the cost performance of the designed solution of the RD strategy by trying to share as many backup resources as possible without modifying the pairing between the RAU nodes and their backup RCC nodes and the routing of the backup connectivity paths. The third strategy, referred to as reconfiguration and improved resource sharing (RIRS), tries to further improve the cost performance of the designed solution of the RD strategy by revisiting the pairing between RAU nodes and their backup RCC nodes, as well as the routing of the backup connectivity path, in such a way that the sharing among backup resources is maximized.

It is worth noting that both PRS and RIRS work in a two-step-fashion. The initial pairing between the RAU node with its primary/backup RCC node as well as the choice for the routing of the primary and backup connectivity paths are done without any consideration on how beneficial such choices are in terms of potential 


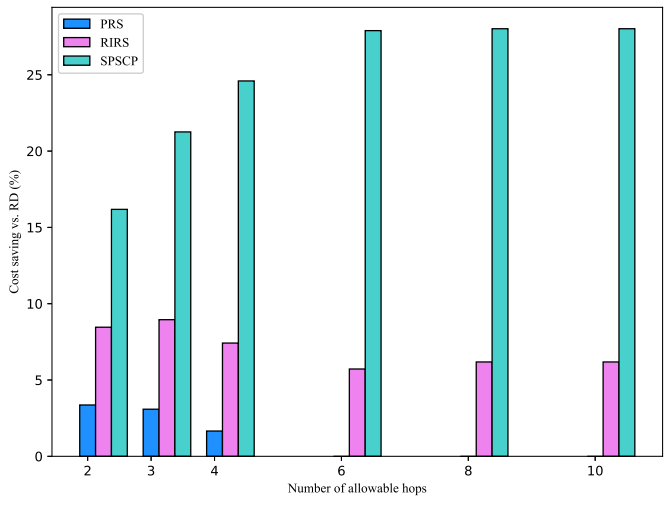

(a) $C_{\text {Conn }}=1, C_{\text {Ser }}=5$

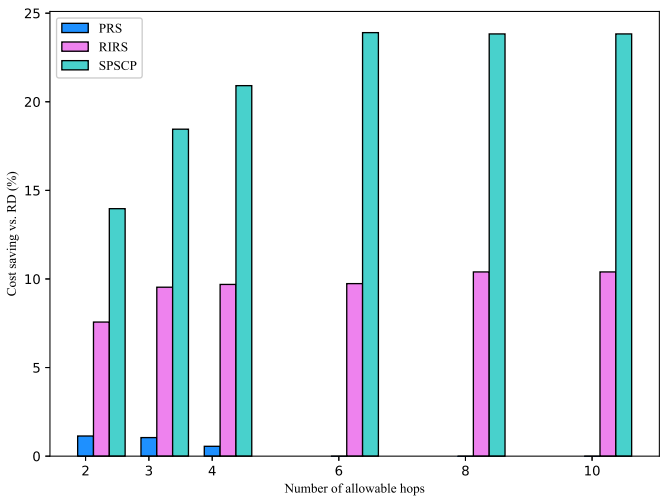

(b) $C_{\text {Conn }}=5, C_{\text {Ser }}=1$

Figure 3: Total cost savings for PRS, RIRS, and SPSCP compared to RD when $C_{R C C}=100$.

shareability of the backup resources. Only at the second stage (i.e., after the initial pairing is completed) backup resources are shared as much as possible. On the other hand, the amount of shareable backup resources is affected by the position of the primary RCC nodes and by the route of their connectivity paths, something that both PRS and RIRS cannot change. Conversely, with SPSCP the shareability potentials of a given primary/backup $\mathrm{RCC}$ node selection and of the route of the primary/backup connectivity path become the primary discriminating factor during the RAU-RCC pairing process.

Statistical results are generated using a large number of independent simulation runs in order to make sure that the confidence interval never exceeds $2 \%$, with a confidence level of $95 \%$.

Fig. 3a shows the cost savings (i.e., percentage with respect to the RD strategy) obtained by the proposed SPSCP solution and by the two benchmark sharing strategies (i.e., PRS and RIRS). The cost gain is reported as a function of the number of allowable hops. The cost of a server unit and of a connectivity unit is 5 [CU] and 1 [CU], respectively. The figure shows that the cost savings of SPSCP vs. RD are between $16 \%$ and $28 \%$. In addition, SPSCP can result in $23 \%$ higher cost savings compared to RIRS for $h \geq 6$. It is interesting to see how the performance of SPSCP improves with higher values of $h$. This is because with a more relaxed hop constraint there are more options for the RAU-RCC pair with good shareability potentials, thus leading to a larger overall gain. On the other hand, with PRS and RIRS we have almost an opposite behavior. PRS and RIRS tend to concentrate RCC nodes in a few midhaul nodes to reduce cost of RCC nodes deployment since they do not consider the potential shareability performance for choosing primary and backup RCC nodes. This in turn limits the amount of backup resources that can be possibly shared.

Fig. $3 \mathrm{~b}$ presents the values of the cost savings when the cost of a server unit and of a connectivity unit is $1[\mathrm{CU}]$ and 5 [CU], respectively. The SPSCP strategy presents similar performance trends with respect to RD, but the difference in cost savings with respect to PRS and RIRS are reduced. These results seem to hint that in the scenario under exam, connectivity resources are the bottleneck of the system and cannot be shared too much. On the other hand, the more backup server resources can be shared the higher are the cost savings.

These speculations are confirmed by the results presented in Fig. 4. The figure shows the breakdown of total design cost, for all four strategies under exam, in terms of primary/backup server costs, primary/backup connectivity costs, and RCC node deployment cost. It is evident from the figure that one of the major factors for cost savings brought by SPSCP is the considerable reduction (i.e., around 50\%) in the number of required backup servers, while the savings in terms of shared backup connectivity resources are negligible. This is the direct result of considering, during the RCC nodes selection process, the shareability potentials of a given primary/backup RCC node selection and of the route of the primary/backup connectivity path.

\section{Conclusions}

The paper presented a cost-efficient solution to design a resilient H-CRAN architecture in a scenario considering failures of servers in the RCC node as well as the nodes/links in the midhaul network. By adopting the proposed strategy, referred to as shared-path shared-compute planning (SPSCP), survivability in the presence of a single failure of these critical resources is guaranteed. SPSCP assigns, to each RAU node, a primary and a backup RCC node with the aim of reducing the overall network design cost. In order to achieve this, the proposed strategy maximizes the number of backup resources (i.e., servers in the RCC nodes and connectivity units in the midhaul network) that can be shared. Simulation results show that SPSCP can yield up to $28 \%$ cost savings compared to the conventional resilient design strategies based on dedicated backup resources. 

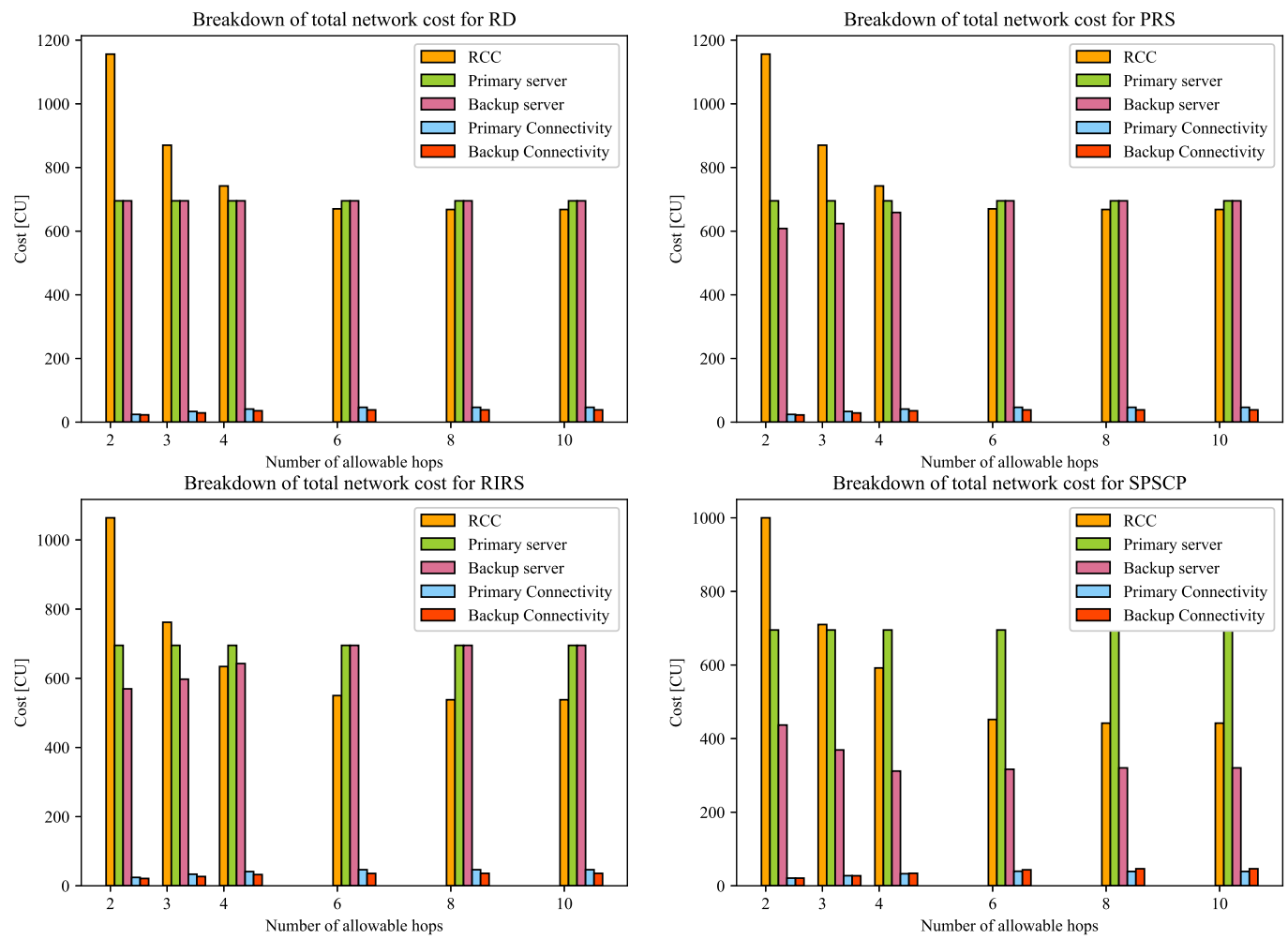

Figure 4: Breakdown of total design cost when $C_{R C C}=100, C_{C o n n}=1, C_{S e r}=5$.

\section{ACKNOWLEDGMENTS}

This work was funded by the MSCA-ITN project 5G STEP FWD with funding from the European Union's Horizon 2020 research under grant agreement number 722429.

\section{REFERENCES}

[1] A. Alabbasi, X. Wang, and C. Cavdar, "Optimal processing allocation to minimize energy and bandwidth consumption in Hybrid-CRAN," IEEE Transactions on Green Communications and Networking, vol. 2, no. 2, pp. 545-555, June 2018.

[2] C. I, Y. Yuan, J. Huang, S. Ma, C. Cui, and R. Duan, "Rethink fronthaul for soft RAN," IEEE Communications Magazine, vol. 53, no. 9, pp. 82-88, September 2015.

[3] “Next Generation Fronthaul Interface," China Mobile Research Institute, Alcatel-Lucent, Nokia, ZTE, Broadcom, Intel, Tech. Rep., June 2015.

[4] A. de la Oliva, J. A. Hernandez, D. Larrabeiti, and A. Azcorra, "An overview of the CPRI specification and its application to C-RAN-based LTE scenarios," IEEE Communications Magazine, vol. 54, no. 2, pp. 152-159, February 2016.

[5] T. RABIA and O. BRAHAM, "A new SDN-based next generation fronthaul interface for a partially centralized C-RAN," in 2018 IEEE 32nd International Conference on Advanced Information Networking and Applications (AINA), May 2018, pp. 393-398.

[6] B. M. Khorsandi, C. Raffaelli, M. Fiorani, L. Wosinska, and P. Monti, "Survivable BBU hotel placement in a C-RAN with an optical WDM transport," in 13th International ConferenceDRCN 2017 - Design of Reliable Communication Networks, March 2017, pp. 1-6.

[7] M. Shehata, F. Musumeci, and M. Tornatore, "Resilient BBU placement in 5G C-RAN over optical aggregation networks," Photonic Network Communications, vol. 37, no. 3, pp. 388-398, June 2019.

[8] M. Y. Lyazidi, L. Giupponi, J. Mangues-Bafalluy, N. Aitsaadi, and R. Langar, "A novel optimization framework for C-RAN BBU selection based on resiliency and price," in 2017 IEEE 86th Vehicular Technology Conference (VTC-Fall), September 2017, pp. 1-6.

[9] B. Han, V. Gopalakrishnan, G. Kathirvel, and A. Shaikh, "On the resiliency of virtual network functions," IEEE Communications Magazine, vol. 55, no. 7, pp. 152-157, July 2017.

[10] "Transport network support of IMT-2020/5G," International Telecommunication Union, Tech. Rep., February 2018. 